\title{
The Interplay Between Mathematicians' Conceptual and Ideational Mathematics about Continuity of Complex-Valued Functions
}

\author{
Hortensia Soto-Johnson $^{1}$ • Brent Hancock ${ }^{1}$. \\ Michael Oehrtman ${ }^{2}$
}

Published online: 7 July 2016

(C) Springer International Publishing Switzerland 2016

\begin{abstract}
Adopting Schiralli and Sinclair's notions of conceptual mathematics (CM) and ideational mathematics (IM), we investigated mathematicians' reasoning about continuity of complex-valued functions. While $\mathrm{CM}$ centers on formal mathematics as a discipline, IM focuses on how an individual perceives formal mathematics. There were four IM notions that the mathematicians used to convey the idea of continuity for complex-valued functions: control, topological features, preservation of closeness, and paths. The mathematicians' IM tended to be grounded in their embodied experiences and espoused for pedagogical reasons, in preparation for other actions, or to assist their own reasoning. Some of the mathematicians' IM metaphors conveyed a domain-first quality, which accounted for the domain of the function before mentioning any objects from the codomain. Given such metaphors did not capture the full structure of the epsilon-delta definition of continuity, the mathematicians transitioned to CM language in an effort to make their IM statements more rigorous. Our research suggests that while IM metaphors stemming from embodied experiences can serve as helpful tools for reasoning about continuity of complex-valued functions, one must be cognizant of ways in which the informal IM must be altered or extended to fully capture the CM. Given the pedagogical intent of many of the participants' domain-first IM examples,
\end{abstract}

Hortensia Soto-Johnson

Hortensia.soto@unco.edu

Brent Hancock

Brent.Hancock@unco.edu

Michael Oehrtman

michael.oehrtman@okstate.edu

1 School of Mathematical Sciences, University of Northern Colorado, Ross Hall 2240C, Greeley, CO 80639, USA

2 Department of Mathematics, Oklahoma State University, MS 426, Stillwater, OK 74074, USA 
we recommend that care be taken during instruction to deliberately elucidate where the IM is incomplete or fails to encapsulate the intricacies of the CM at hand.

Keywords Complex-valued functions · Conceptual mathematics · Continuity - Ideational mathematics · Mathematicians

\section{Introduction}

Some empirical studies have explored students' understanding of continuity of functions from $\mathbf{R}$ to $\mathbf{R}$, and a few linguistic and theoretical investigations have explored mathematicians' reasoning about these ideas. To extend these findings we explored the relationships between mathematicians' use of standard forms of mathematical communication and their personal reasoning about continuity of complex-valued functions, from $\mathbf{C}$ to $\mathbf{C}$ or equivalently from $\mathbf{R}^{\mathbf{2}}$ to $\mathbf{R}^{\mathbf{2}}$. We adopted Schiralli and Sinclair's (2003) framework of conceptual mathematics (CM) and ideational mathematics (IM), where $\mathrm{CM}$ refers to the publicly accessible tools and shared (although imperfect) meanings negotiated within the mathematics community and IM refers to how individuals perceive those mathematical tools and concepts. In this report we address the question: What is the nature of mathematicians' conceptual and ideational mathematics regarding continuity of complex-valued functions? As part of this research question we also explored the interplay between mathematicians' CM and IM for this mathematical topic with the goal of extending theory to include the nature of the interaction between experts' CM and IM.

Our findings suggest that the mathematicians weave in and out of the domains of $\mathrm{CM}$ and IM in purposeful and integrated ways. As discussed in the "Theoretical Perspective" section, although Schiralli and Sinclair (2003) view embodied mathematics as the underlying source of both CM and IM, it is not obvious how CM and IM interact in practice. Our work explores how and why experts might navigate among $\mathrm{CM}$ and IM reasoning, and to strengthen the IM/CM theory in doing so. We found that choices for navigating from conceptual to ideational mathematics included pedagogical reasons, preparation for other explanations, or to assist the mathematicians' own reasoning. The mathematicians transitioned from ideational to conceptual mathematics language in an effort to portray more rigorous explanations or in preparation for other explanations. Furthermore our participants tended to incorporate IM in the form of metaphors based on their physical embodied experiences in an attempt to convey CM regarding continuity of complex-valued functions. Some of these IM explanations were domain-first conceptualizations, containing inconsistencies with the CM epsilon-delta definition of continuity. We believe that this research contributes to the growing body of literature on mathematicians' reasoning about mathematical topics (Anderson and Leinhardt 2002; Inglis and Alcock 2012; Martin 2013; Nemirovsky and Smith 2011; Sinclair and Tabaghi 2010; Weber 2008; Weber and Mejia-Ramos 2011; WilkersonJerde and Wilensky 2011), and may inform the teaching of continuity of real-valued functions, for which there is minimal research (Fisher 2010; Oehrtman 2009). Finally, our research may inform the teaching and learning of complex analysis. Before elaborating the notions of CM and IM and describing our participants, we summarize some of the literature relevant to complex numbers, limits, continuity of real-valued functions, and mathematicians' reasoning about mathematics. 


\section{Literature Review}

\section{Complex Numbers and Variables}

The literature on teaching and learning of complex numbers, variables, and functions is minimal and tends to center on undergraduates' understanding of the arithmetic of complex numbers. In general, researchers (Danenhower 2000, 2006; Panaoura et al. 2006) document that undergraduates demonstrate a static view of the arithmetic of complex numbers and do not navigate effectively between algebraic and geometric representations of complex numbers and complex-valued equations. Teaching experiments (Harel 2013; Nemirovky et al. 2012; Soto-Johnson and Troup 2014) have demonstrated students can develop a dynamic view of the arithmetic of complex numbers. Nemirovky et al. (2012) presented promising tactics for developing preservice and inservice secondary teachers' geometric interpretations of the arithmetic of complex numbers. Not all research related to the teaching and learning of complex numbers has been conducted with undergraduates and in-service teachers; some research includes high school students. For example, Soto-Johnson (2014) illustrated how technology assisted high school students to view the arithmetic operations of complex numbers as transformations of the complex plane. In another study, Panaoura et al. (2006) investigated Greek high school students' ability to navigate between algebraic and geometric representations of complex-valued equations and inequalities of the form $\left|z-z_{0}\right| \leq k$, but their findings also suggest that the students tended to compartmentalize symbolic/algebraic, geometric, and verbal representations.

\section{Limits}

Limit definitions of continuity by nature first characterize continuity at a point. Students tend to find this notion nonsensical when they first encounter it and typically reject the possibility that a real-valued function may be continuous only at a single point. Nonetheless, many students' interpretations of limits are similar to those for continuity. Williams (1991) administered a survey to 341 students in a second semester calculus course to determine the predominance of standard interpretations of limits and conducted follow-up interviews with 10 students to characterize the details of their thinking and learning. Williams found that students viewed counterexamples as minor exceptions rather than reasons to reformulate an incomplete or problematic concept, resulting in highly resilient misconceptions. The students also ubiquitously relied on dynamic imagery, limits being unreachable, and a "generic metaphor" (p. 234) of limits based on the presumption of access to an accurate and detailed graph.

Through a cognitive linguistic analysis, Lakoff and Núñez (2000) outlined a series of metaphorical maps on which an expert may ground their conception of limits in "embodied experience." They argued that a "basic metaphor of infinity" forms the foundation of such a development where experiences with completed iterative processes serve as a foundation for reasoning about never-ending iterative processes. By transferring relationships and implications of a final state in a completed process to a final "infinite" state, Lakoff and Núñez showed that one may conceive of limits for monotonic sequences, general sequences, and functions of real numbers. Shifting the lens from mathematicians' to students' metaphorical reasoning, Oehrtman (2009) 
collected writing samples from 120 students enrolled in a year-long calculus sequence and conducted interviews with 20 of these students. Using Max Black's $(1962,1977)$ characterization of ontologically creative metaphors as those involving both emphasis (commitment by the speaker) and resonance (sufficient structure to support implicative elaboration), Oehrtman identified five strong metaphor clusters based on objects, relationships, and logic related to a collapse in dimension, approximation and error analysis, proximity in a space of point-locations, a small physical scale beyond which nothing exists, and the treatment of infinity as a number. On the other hand, students' reasoning about motion, zooming, and language involving "arbitrarily" and "sufficiently" small quantities failed to meet criteria of either or both emphasis and resonance.

\section{Continuity}

Since concepts of continuity predated those of limits, it is instructive to explore natural interpretations of continuity that arose independently. Many students come to view the formal epsilon-delta definition as "elusive and counterintuitive" (Núñez and Lakoff 1998 , p. 85) since it is intended to resolve technical issues that they have not typically encountered. When students' concept images for continuity dictate that a function should not have any gaps in its graph, should be "all in one piece," and should have "a single formula," then their perceptions of continuity become problematic in more "exotic" scenarios (Tall and Vinner 1981). For instance, if the domain of a function is altered to include only rational numbers, then one can construct continuous functions which conflict with all of the aforementioned concept images.

Accordingly, Núñez and Lakoff (1998) investigated continuity through a cognitive linguistics lens, making a distinction between "natural" continuity and "formal" continuity. Specifically, natural continuity is historically based on trajectory of motion, where this motion is unimpeded by gaps or interruptions. In particular, natural continuity incorporates conceptual metaphors such as fictive motion (Talmy 1996), a cognitive mechanism permitting one to conceptualize a static object in terms of hypothetical motion, such as the motion of tracing a curve. Thus, individuals often express mathematical concepts through dynamic language such as "the function blows up at zero" or "the limit as $x$ approaches zero." From this perspective, a function such as $f$ in Fig. 1a is not "naturally continuous," since it does not represent a possible trajectory of motion. As such, Núñez and Lakoff tracked the introduction of metaphors of gaplessness (for lines) and preservation of closeness (for functions) as discussed by Dedekind and Weierstrass. This was an effort to conceptualize continuity in a new and fundamentally different light, while simultaneously fostering mathematical formalization.

As the previous example illustrates, these new metaphors not only suppress the dynamic nature of conceptions of continuity, but also support a fundamentally different version of the concept. For example, Oehrtman (2009) found that when reasoning about limits and continuity, students invoked numerous dynamic images but rejected notions of objects moving. Even if they allowed for motion, students' reasoning about continuity was a result of the underlying topographical features of the graph, e.g., whether it had a hole or cliff. Further complicating the issue, the various metaphors at the foundation of formalization are not even entirely compatible with one another. For example, preservation of closeness is often expressed in terms of a condition of not tearing or introducing gaps in the graph. Formulating such gaps topologically, one may 


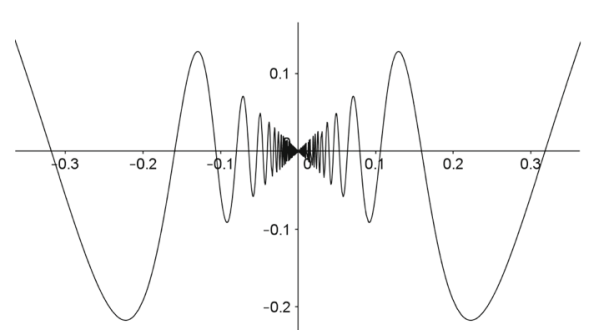

a) $f(x)=\left\{\begin{array}{cc}x \sin \frac{1}{x}, & x>0 \\ 0, & x \leq 0\end{array}\right.$

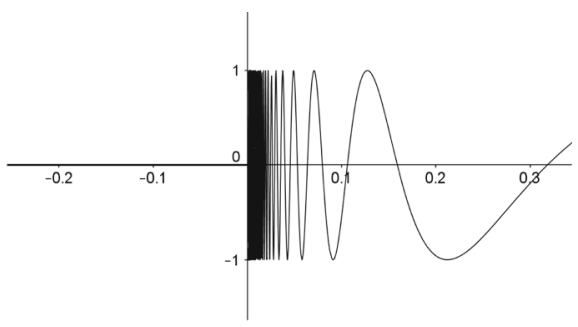

b) $g(x)=\left\{\begin{array}{cc}\sin \frac{1}{x}, & x>0 \\ 0, & x \leq 0\end{array}\right.$

Fig. 1 "Natural continuity" differs from Weierstrass continuity: a) a continuous function $f$ whose graph does not represent a path of motion, b) a discontinuous function $g$ which preserves gaplessness

arrive at the properties that continuous functions must preserve both connected and path-connected sets. However, neither of these properties are sufficient to infer Weierstrass' notion of continuity because functions such as $g$ in Fig. 1b preserve both types of connectedness. Thus the graphs are "gapless" in this sense yet the function does not preserve closeness. Additionally, while the purely topological formulation of continuity (the preimage of an open set is open) is inspired by the underlying epsilondelta interpretation, it extends the notion to non-metric spaces where preservation of closeness is an even more problematic conception.

\section{Mathematicians Reasoning about Mathematics}

Investigating and comparing mathematicians' and students' reasoning about mathematical concepts has been a growing area of research and can serve as a mechanism for "discerning and understanding the development of desirable ways of thinking, particularly those that brand certain mathematical areas" (Harel 2013, p.16). A majority of the research exploring mathematicians' mathematical reasoning focuses on proof validation (Inglis and Alcock 2012; Weber 2008; Weber and Mejia-Ramos 2011) and indicates that mathematicians tend more to the logical structure of a purported proof while students tend to focus on surface features of a purported proof. Furthermore, as mathematicians validate purported proofs, they appear to use a variety of modes of reasoning such as formal reasoning, informal deductive reasoning, example-based reasoning, and rigorous proof construction (Weber 2008).

Other researchers have explored how mathematicians convey mathematical notions such as projections (Anderson and Leinhardt 2002), eigenvectors (Sinclair and Tabaghi 2010), pointwise convergence in a Taylor series (Martin 2013), and topological notions (Nemirovsky and Smith 2011; Wilkerson-Jerde and Wilensky 2011). As with other research that compares mathematicians' and students' reasoning, Martin also showed that mathematicians tend to integrate conceptual images, while novices concentrate on surface level features. Specifically, formal theory guided mathematicians' conceptual images and navigation between their various images. Sinclair and Tabaghi also illustrated how mathematicians' conceptual understanding of eigenvectors informed their ideational understanding. This conceptual understanding allowed mathematicians to create dynamic metaphors associated with the static algebraic representations. Furthermore, mathematicians conveyed the dynamic aspects of their metaphors via diagrams 
and gestures. Nemirovsky and Smith (2011) argued that such gestures signal relationships between symbols and diagrams. Wilkerson-Jerde and Wilensky (2011) claimed mathematicians navigate their concept images intentionally in an effort to deconstruct and coordinate substructures of mathematical objects at hand.

\section{Theoretical Perspective}

Our theoretical perspective falls under the umbrella of embodied cognition, which has various interpretations. Following other researchers (Châtelet 2000; de Freitas and Sinclair 2012; Nemirovky et al. 2012) we view learning and experience within an environment as inherently inseparable. For us, knowing is doing, and is observable. The observable aspect of knowing can manifest itself in various ways including "bodily actions taken within the physical environment for the purpose of doing mathematics and reasoning about mathematics, which we assume is based on our participants' subjective experience with their world" (Soto-Johnson and Troup 2014, p. 112). Such knowledge can be conveyed verbally, in writing, via enactments such as gesture and formally or informally, which Schiralli and Sinclair (2003) capture with the terms conceptual mathematics (CM) and ideational mathematics (IM).

Schiralli and Sinclair (2003) supplemented Lakoff and Núñez (2000) embodied cognition framework to include not only the use of metaphor but also how metaphors are created. In this lens, Schiralli and Sinclair stress the importance of analyzing the meaning behind thinking abstractly and how this affects one's use of metaphors depending on whether one is "learning, doing or, using mathematics" (p. 81). In order to extend the belief that abstract mathematical concepts are grounded through a combination of metaphors and sensory-motor experiences, the researchers dichotomized mathematical understanding into CM and IM. They defined CM as "mathematics as a subject-matter or discipline" (p. 81) whereas, IM indicates how one perceives or thinks about mathematical concepts. CM is accessible within the public domain of the mathematics community, and is idealized in mathematical communication such as formal presentations, research papers, and textbooks. Individuals may interpret CM differently and the breadth of those interpretations constitutes one's IM.

According to Schiralli and Sinclair (2003) someone engaged in mathematical rules, operations, and concepts is on the path to learning CM. It is during this engagement that IM and CM become intimate and less of a dichotomy, but navigating from IM to CM generally entails untutored mathematical awareness (UMA) developed by partaking in everyday activities. While UMA and IM facilitate developing existing CM, it is possible that they also contribute to propositions, creations, or inventions of new CM. Furthermore, we believe that one's exposure to CM combined with one's culture, past experiences, and mathematical background can influence one's IM. Schiralli and Sinclair exemplified embodied mathematics as the foundation for the interplay between UMA, IM, and CM and may account for the creation of a metaphor. They elaborate how embodied mathematics "... may be understood as the embodiment - the embodied apprehension - of ordering principles and categories the human organism has/acquires ... beneath the level of conscious awareness but which variously is involved in all other senses ..." (p. 87). An individual's viscera, senses, and interaction with a mathematical concept may lead to the simultaneous construction of a metaphor for the concept. The attention to such metaphor 
constructions is what distinguishes the IM perspective from Lakoff and Núñez (2000) embodied cognition framework. In order to explore how learners craft metaphors and how sensory-motor experiences may contribute to such creations, Schiralli and Sinclair called for empirical studies "in which these sensory-motor experiences might variously be structured by those common neuro-physiological predispositions human beings genetically possess and might variously be mediated by environmental factors including those developing cultural and symbolic systems" (p. 90).

We view an individual's development of IM and CM as occurring through the dialectic interplay between one's application of embodied concepts while reasoning about a mathematical task and his/her attention to the constraints and needs of the formal logical system. In one of his formulations of the zone of proximal development (ZPD), Vygotsky (1987) similarly framed the interaction between everyday and scientific concepts. From his work, one may infer that the strengths of one's everyday concepts (spontaneous application, concrete meaning, and effective reasoning within familiar settings) are weaknesses for the scientific, but the strengths of one's scientific concepts (volitional application, abstract interpretation, and effective reasoning about systematic relationships) are weaknesses for the everyday concepts. We adopted the $\mathrm{CM}$ /IM relationship as a theoretical framework precisely to explore how such strengths and weaknesses of CM/IM reasoning informs one's IM/CM reasoning. This framework was especially useful because our participants were mathematicians who we assumed had developed richly integrated CM and IM reasoning about continuity of complexvalued functions. As such, our data collection, analysis, and interpretation were designed to capture the interplay between $\mathrm{CM}$ and IM reasoning.

\section{Methods}

\section{Participants and Setting}

This report is part of a larger study where we explore mathematicians' geometrical understanding of the arithmetic of complex numbers and analysis of complex-valued functions. Five mathematicians from three different institutions served as our participants and participated in a 90-min video-taped interview. Becky, Judy and Rafael's area of expertise is complex analysis, Luke's area of expertise is differential geometry, and Andrew's is differential equations (all psuedonyms). In this report, we focus on the responses to the continuity item, which was stated as: In calculus, we sometimes use the idea of tracing the graph of a function and not lifting our pencil to convey the concept of continuity. What geometric representation or explanation might be useful to understand continuity of complex-valued functions? We also presented the participants with two complex-valued functions, $f(z)=\frac{\operatorname{Re}(z)}{|z|}$ and $f(z)=\frac{z \operatorname{Re}(z)}{|z|}$, and asked them to determine whether the functions could be extended to make them continuous at $z=0$. The first prompt was open-ended to elicit either CM or IM reasoning, but also to remind the participants of a typical geometric explanation used to explain continuity of real-valued functions. We anticipated that the first question would elicit IM reasoning backed by rich $\mathrm{CM}$ and that the tasks involving the two functions would elicit $\mathrm{CM}$ reasoning backed by rich IM. As the participants responded to the prompts we probed 
in an effort explore the mathematicians' $\mathrm{CM}$ and IM and the interplay between them related to continuity of complex-valued functions.

\section{Concept Analysis}

Before detailing our analysis techniques and results, we provide a formal definition of continuity of complex-valued functions which states: "for each positive number $\varepsilon$, there is a positive number $\delta$ such that $\left|f(z)-f\left(z_{0}\right)\right|<\varepsilon$ whenever $\left|z-z_{0}\right|<\delta$. A complex-valued function is said to be continuous in a region $R$ if it is continuous at each point in $R$ " (Brown and Churchill 2009, p. 53). Given the theorems that follow from this definition, there are various ways to convey all or parts of this definition. For example one may discuss preservation of closeness or violation of preservation of closeness, by exploring the image of various portions of the domain. Continuity of complex-valued functions may also be discussed in terms of multivariable calculus, where one recognizes that the function is continuous if and only if the functions making up the real and imaginary components are continuous. One might also consider the continuity of a function by investigating the limit as one approaches the point $z_{0}$ via different paths.

Continuity of complex-valued functions depends on the notion of a limit of a function at a point in the interior of its domain in $\mathbf{R}^{\mathbf{2}}$. We highlight two equivalent definitions of this concept. Let $f: \mathbf{R}^{2} \rightarrow \mathbf{R}^{2}$ be a given function defined at all points $x$ in some deleted neighborhood of a point $x_{0}$. Then $\lim _{x \rightarrow x_{0}} f(x)=L$, if, for all $\varepsilon>0$, there exists a $\delta>0$ such that $|f(x)-L|<\varepsilon$ whenever $0<\left|x-x_{0}\right|<\delta$. This definition for multivariable functions is often characterized in terms of limits of sequences or paths with simpler domains. Specifically $\lim _{x \rightarrow x_{0}} f(x)=L$, if for every sequence $\left(a_{n}\right)$ in $\mathbf{R}^{\mathbf{2}}$ with $\lim _{n \rightarrow \infty} a_{n}=x_{0}$, we must have $\lim _{x \rightarrow \infty} f\left(a_{n}\right)=L$. Likewise, $\lim _{x \rightarrow x_{0}} f(x)=L$ if for every path $\gamma:(0,1) \rightarrow \mathbf{R}^{2}$ with $\lim _{t \rightarrow 0} \gamma(t)=x_{0}$, we must have $\lim _{t \rightarrow 0} f(\gamma(t))=L$. These limit definitions then give rise to corresponding continuity characterizations for continuity of the function $f$. Specifically, $f$ is said to be continuous at the point $x_{0}$ if $\lim _{x \rightarrow x_{0}} f(x)=f$ $\left(x_{0}\right)$, where this limit can be conceived of in any of the above three ways. A function is continuous on a set in its domain if it is continuous at every point in that set. Converting the inequalities $\left|f(x)-f\left(x_{0}\right)\right|<\varepsilon$ and $\left|x-x_{0}\right|<\delta$ to basis disks for the topology of $\mathbf{R}^{\mathbf{2}}$, one may also arrive at the definition that a function $f$ is continuous on a domain if the preimage of every open set is open in that domain.

There are several ways in which continuity can be violated. In particular, $f$ has a removable discontinuity at $x_{0}$ if $\lim _{x \rightarrow x_{0}} f(x)$ exists but $\lim _{x \rightarrow x_{0}} f(x) \neq f\left(x_{0}\right)$. Alternatively, if $f$ has a nonremovable discontinuity at $x_{0}$, the limits along all paths approaching $x_{0}$ may exist but differ or there may be some paths approaching $x_{0}$ such that $\lim _{x \rightarrow x_{0}} f(x)$ does not exist. If $f: C \rightarrow C$ is a complex-valued function then we can write $f$ in terms of its real and imaginary components, i.e., $f(z)=u(x, y)+i v(x, y)$ where $u: \mathbf{R}^{2} \rightarrow \mathbf{R}$ and $v: \mathbf{R}^{2} \rightarrow$ $\mathbf{R}$. Then $f$ is continuous if and only if its component functions, $u$ and $v$, are continuous.

In light of the above definitions, we now discuss two related ideas commonly associated with continuity: preservation of closeness and ripping. Preservation of closeness is the notion that if two points located in the domain of a function are "close" 
then they will end up "close" under the image of that function. The negation of the $\varepsilon-\delta$ continuity definition requires an $\varepsilon>0$ such that for every $\delta>0$, there exists some $z$ such that $\left|z-z_{0}\right|<\delta$ and $\left|f(z)-f\left(z_{0}\right)\right|>\varepsilon$. In particular, the latter half of this negation indicates that some points within a distance of $\delta$ from $z_{0}$ get mapped at least a distance of $\varepsilon$ from $f\left(z_{0}\right)$ and therefore preservation of closeness does not hold. If the domain is imagined to be made of a physical material and the mapping is conceived as trying to simultaneously stretch every point in the domain to its image, violating preservation of closeness would result in ripping the material.

\section{Analysis}

Our first step in analyzing the data was to transcribe all verbiage from the interviews and partition each transcription into short episodes for the purpose of coding. As part of the transcription we also documented algebraic and geometric inscriptions that the participants wrote on the board along with descriptions of gestures made by the participants. Such notes allowed us to examine the various mechanisms used by the participants to communicate their CM and IM in relation to continuity. Each researcher took general notes on each episode while watching a subset of the video data. Upon reviewing all video footage and the corresponding notes as a group, we inductively established codes (see Appendix) until we reached an exhaustive list. We then assigned these codes to each portion of each participant's transcript and coded each episode as primarily IM or primarily CM. To do this, we turned to participants' verbiage that indicated whether they were conveying IM or CM. For instance, IM statements often expressed personal or pedagogical preferences or tendencies when thinking about a topic, and began with verbiage such as "I tend to think about [...]" or "I often end up describing this as [...]." On the other hand, CM statements often began with verbiage such as, "But to be more precise [...]" or "Speaking more mathematically [...]." For example, Judy discussed paths of approach in one instance as IM: "I often end up describing the difference between driving on a single lane road and being on a big wide open parking space, with people driving in all different directions" But, elsewhere Judy invoked CM to describe paths: "you can approach the origin from different directions and get different heights on the $x-y$ plane." There were also episodes that we coded as $\mathrm{CM}$ based on our knowledge of what is generally accepted in the larger mathematical community. However, not all statements were easily identified as IM or CM; we discussed these cases as a group until we came to a consensus. Sometimes participants also used language, inscriptions, or gestures in one domain to refer to a concept from the other domain. We documented these instances to aid our analysis of participants' transitions from IM to CM, and vice versa. Finally, we identified particular instances during each interview where there was a clear reason for a participant instantiating IM or $\mathrm{CM}$ and we refined our coding to incorporate this information.

Once we garnered this information, we summarized our findings for each participant in a "timeline," indicating his/her CM and IM, the interplay between these notions, and the rationale for each instance of CM and IM, where applicable. The timelines also have the potential to highlight surprises. For example, given the nature of the tasks, one might hypothesize that participants will begin with IM responses and navigate towards $\mathrm{CM}$ responses. Our data indicate that this is not the case and in fact, the participants tended to frequently navigate between the two modes. Although the timelines indicate 
the approximate time for each piece of the participants' responses, we are not interested in the change of CM/IM over time. Rather, the time-stamps helped us document how long a participant conveyed IM or CM notions and most importantly they helped us illustrate how and why a participant navigated between IM and CM.

\section{Results}

In this section we provide a summary of each of the participants' responses along with their timeline depicting the various topics conveyed by the participant. The timeline gives the reader a feel for how long (in minutes) a participant spent on a topic and an indication of how and why the participant navigated between CM and IM. Each rectangle in the figure contains the code word illustrating how the participants expressed their perceptions using either CM or IM language. When a participant explicitly expressed a reason as to why they were conveying ideas in a given format, we documented their reasoning underneath the corresponding conceptual and ideational codes. The arrows in the figure indicate instances when a participant used language in one domain to express a concept from the other domain. For example, an arrow from an IM rectangle to a CM rectangle meant that the participant used IM language to express a CM notion. The reader should note that in some cases we do not discuss the contents of each rectangle due to the length of the response. On the other hand, we attempted to capture all the notions found in the figure as part of a summary for each participant's response.

\section{Andrew}

Compared to the other participants, Andrew's responses were primarily expressed in CM language (Fig. 2). He stated that we cannot construct an analogy corresponding to lifting one's pencil off the page to describe continuity for complex functions, because their graphs are four-dimensional, which we cannot draw in the traditional sense (Fig. 2a). Andrew invoked $\mathrm{CM}$ as he discussed the component functions; that is, $f$ : $C \rightarrow C$ where $f=u+i v$ such that $u: \mathbf{R}^{2} \rightarrow \mathbf{R}$ and $v: \mathbf{R}^{2} \rightarrow \mathbf{R}$ (Fig. 2b), to characterize continuity in terms of his IM of preservation of closeness for pedagogical purposes (Fig. 2c). He remarked, “And in a sense, especially if you're just trying to explain, then

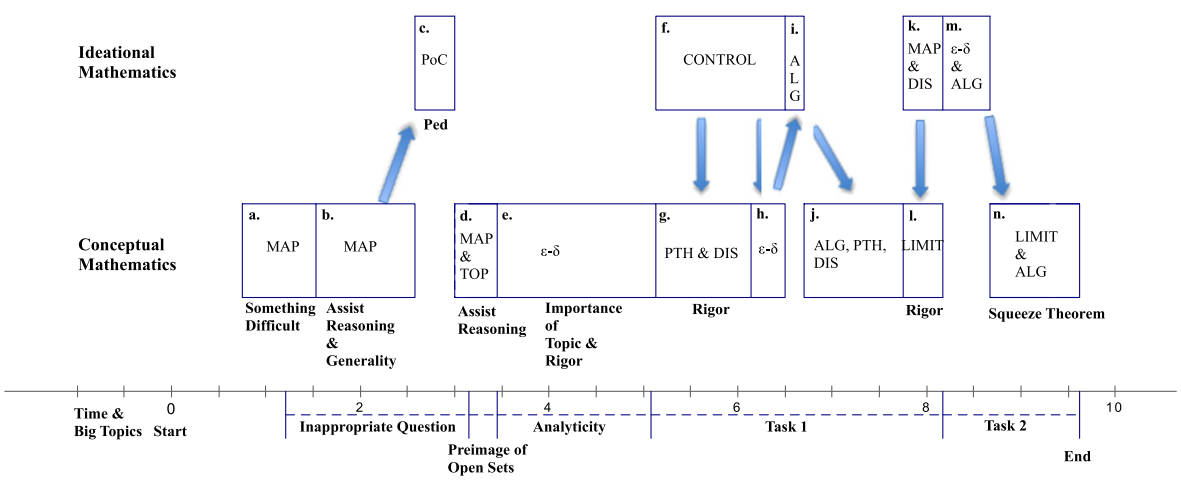

Fig. 2 Andrew's timeline 
you can go here and sort of explain the calculus. You explain that [the graph of $u$ ] doesn't have any gaps and [the graph of $v$ ] doesn't have any gaps." He transitioned back to $\mathrm{CM}$ as he explained how he would depict a function as a mapping from one plane to another plane and would describe continuity topologically. In other words, he would determine if the preimage of an open set is open (Fig. 2d). Andrew was the only participant who noted that analyticity of a function, not continuity, is the central idea for a complex analysis course, but that this definition requires similar $\varepsilon-\delta$ characterizations (Fig. 2e).

While working with the first function, Andrew articulated IM language about various types of "control" to describe his reasoning about limits and continuity. His first use of control acknowledged a need to account for limits along all possible paths that approach a given point (Fig. $2 \mathrm{f}-\mathrm{g}$ ).

Since your domain is two-dimensional we need to control all possible ways of approaching a point. Because there are examples where you have this limit [approaching from right] and this limit [approaching from left] (Fig. 3a) or this limit [approaching at a 45 and $135^{\circ}$ angle] is the same and this limit [spiral] (Fig. 3b) is different.

Andrew also described various types of control that he imagined for the $\varepsilon-\delta$ definition: "The way to think about limits - you want to bound [the radius of the disk in the domain] by absolute value and control that" (Fig. 2h). This need to control the size of a disk in the domain (implicitly to control the magnitude of the function since the limit along some paths is 0) led Andrew to rewrite parts of the algebraic expression in polar coordinates (Fig. 2i). Reviewing this algebraic form (Fig. 3c), he quickly concluded that limits along different paths could be different and the function could not be made continuous at the origin (Fig. 2j). Apologizing for the lack of rigor in this argument, he invoked the limit definition of continuity (Fig. 2k). Figure 3 contains illustrations of Andrew's response to task 1.

In working through the second task, Andrew clarified why this control in the domain is necessary: "Because you're throwing in an extra $r, z x / r$, so the moment you can bring this thing in [the extra $r$ ] you start controlling [the magnitude of the function] uniformly" (Fig. 21). He then expressed this algebraically, and concluded "What I'm trying to say is if you look at the absolute value of that expression over there $[z \operatorname{Re}(z)$ / $|z|]$, it is less than or equal to $r$. I just used the squeeze theorem" (Fig. 2m).

In summary, Andrew discussed CM concepts of: the graph of a function, component functions from $\mathbf{R}^{\mathbf{2}}$ to $\mathbf{R}$, mapping from a plane to a plane, point-wise mapping, continuity as the preimage of open sets being open, analyticity, absolute value, approaching along different paths, the limit of a function, and the Squeeze Theorem. His IM responses included ideas of gaplessness and the notion of control. It also appeared

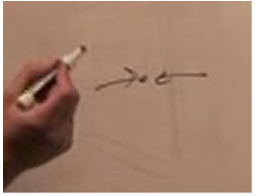

a) Andrew approaching a point from left and right

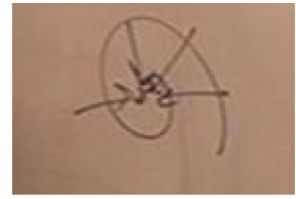

b) Andrew approaching a point via a spiral

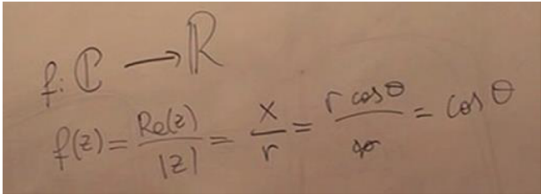

c) Andrew's algebraic approach to task 1

Fig. 3 Andrew's explanation to determining whether the function $f(z)=\frac{\operatorname{Re}(z)}{|z|}$ can be redefined at $z=0$ so that $f$ is continuous 
that Andrew used his IM of control to support the CM notion of uniform convergence in his limit discussion.

\section{Becky}

Becky's timeline (Fig. 4) illustrates her immediate use of CM as she articulated that she thinks about functions as mappings that act on points in the plane (Fig. 4a). She indicated a preference to discuss continuity by contrasting it with a discontinuous function such as $f(z)=\frac{1}{z}$ (Fig. 4b-c). Becky described how she imagined $f$ mapping a disk of radius 2 to the region outside of a circle with a radius of $1 / 2$. Using CM topological language, she commented how "the boundary bits go to the boundary bits." Moving to IM language (Fig. 4d-e) she expressed how zero going off to infinity illustrated the discontinuity because "stuff near [the origin]... goes to drastically different places in the plane." She illustrated this by sketching a "little curve" through the origin in the domain and its image in the codomain, as a split curve in the first and third quadrant (Fig. 5). It is difficult to see the split curve in the figure, because she lightly indicated the curves with a marker.

Before starting the tasks, Becky suggested perceiving discontinuity as ripping a piece of silly putty (silicone-based substance that stretches), which is a physically conceived violation of preservation of closeness (Fig. 4f).

An analogy of not lifting up the pen is if you made a region out of silly putty, and you applied the function to every point in that region, what would that shape look like? Would you have to rip the silly putty to get there? So that's how I think of discontinuity.

Becky framed her discussion of the first task, $f(z)=\frac{\operatorname{Re}(z)}{|z|}$, using CM by rewriting the function in Cartesian form as $\frac{x}{\sqrt{x^{2}+y^{2}}}$ and recognizing that the real valued numerator is simply re-scaled, thus the function maps everything to the real line (Fig. 4g). By approaching $z=0$ via different paths, Becky recognized that the function would "fall apart at zero" and relayed this image to her silly putty metaphor (Fig. 4h-i), "so again if the plane was made out of silly putty I am going to turn it inside out and there is a rip at the origin." She concluded her discussion of the first task using CM and commented that there was not a removable discontinuity (Fig. $4 \mathrm{~m}$ ).

Becky claimed she used algebraic reasoning with the second task, $f(z)=\frac{z \operatorname{Re}(z)}{|z|}$, because it helped her to determine the behavior of the function. This resulted in Becky
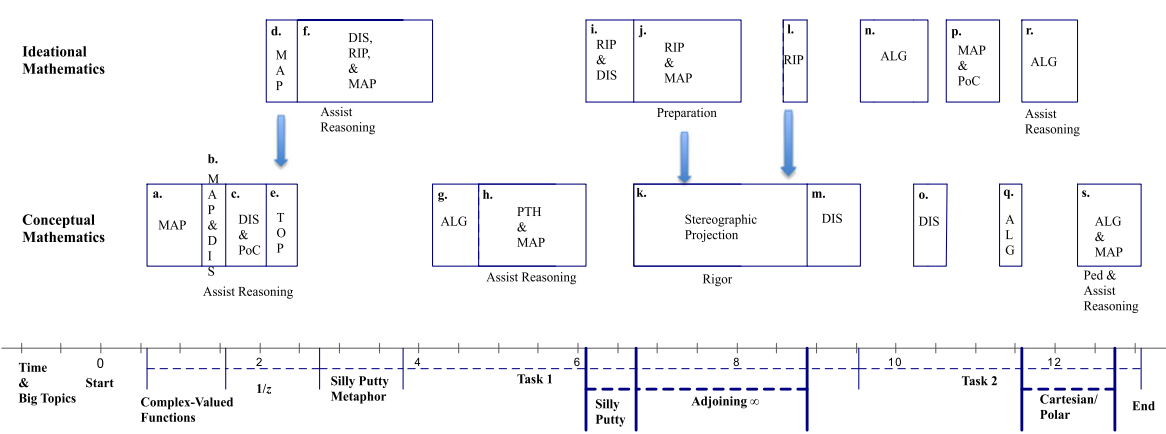

Fig. 4 Becky's timeline 


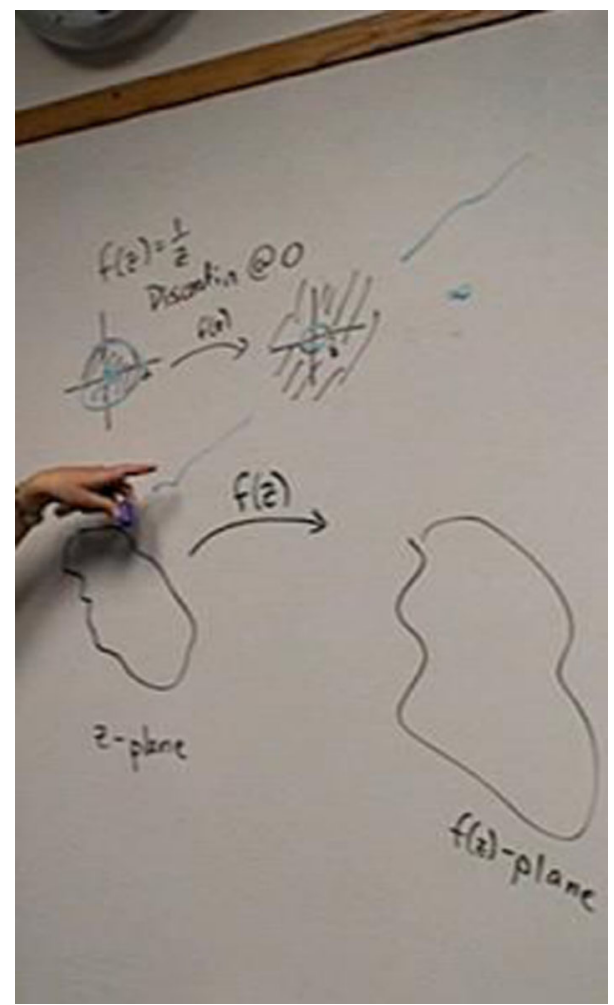

Fig. 5 Becky's explanation of why $f(z)=\frac{1}{z}$ is discontinuous at $z=0$

navigating between IM and CM explanations (Fig. 4n-s). For example, after rewriting the function as $\frac{z^{\frac{1}{2}}}{(\bar{z})^{\frac{1}{2}}} \operatorname{Re}(z)=\left(\frac{z}{\bar{z}}\right)^{\frac{1}{2}} \mathbf{R e}(z)$ Becky wanted to show that "stuff near zero . . gets mapped to zero." Laughing, she expressed how thinking about the factor $\operatorname{Re}(z)$ in Cartesian form and the factor, $\frac{z^{\frac{1}{2}}}{(\bar{z})^{\frac{1}{2}}}$ in polar form was easier for her because $\operatorname{Re}(z)$ " picks off something Cartesian" and $\left(\frac{z}{\bar{z}}\right)^{\frac{1}{2}}$ computes a modulus which "really wants [her] to think in polar." This perception allowed her to recognize that points close to zero "spin around" and thus she could redefine the function to make it continuous at zero (Fig. 4s).

After completing the tasks, Becky expressed that she tends to think about mathematics algebraically rather than graphically, but her algebraic discussion integrated both $\mathrm{CM}$ and IM and revealed a dynamic nature of her concept of continuity. By observing the timeline one see that Becky discussed CM ideas concerning: Cartesian and polar coordinates, stereographic projection, compactification, discontinuity, paths approaching the origin, collapsing to the real line, the point-wise behavior of a mapping, and the boundary of a region. IM ideas included: the image of paths getting split up, preservation of closeness, ripping or tearing silly putty, "spinning things around," and combining the Cartesian and polar representations into what she referred to as "the blend." Although Becky navigated between CM and IM in an effort to assist her own reasoning, some instances of CM seemed to serve a pedagogical purpose. For example, 
she expressed how she would blend the Cartesian and polar representations for herself, but would not explain such a solution to students because they might find it confusing. It is noteworthy that Becky did not discuss approaching a point via different paths until she addressed the continuity of the two functions.

\section{Judy}

Figure 6 depicts Judy's response, which began with primarily IM language, where she indicated that the analogy of "not lifting your pencil" is useful, but that in order to be more rigorous, one has to realize that in two dimensions there are more paths to consider when approaching a given point (Fig. 6a). Judy explained that in the case of a real-valued function, the analogy tends to capture nearly all of the examples of a discontinuous function, in which "you would have to hop off of your page" (Fig. 6b). In discussing the pedagogical transition from teaching limits in $\mathbf{R}$ to teaching limits in $\mathbf{C}$, Judy noted,I often end up describing the difference between driving on a single lane road and being on a big wide open parking space, with people driving in all different directions. There's just a lot more going on, and a lot of other directions ... where you might be making approaches" (Fig. 6c-d).

Transitioning back to CM, Judy explained that when considering continuity near the origin, one has to consider all possible paths that approach the origin (Fig. 6e). Judy pointed out that she sometimes prefers to think of complex-valued functions as a map from $\mathbf{R}^{2}$ to $\mathbf{R}^{2}$, even though "it's hard to visualize two dimensions to two dimensions" (Fig. 6f). She outlined two ways of thinking about complex-valued functions. Judy's first method entailed a sketch to indicate a mapping from one plane to another (Fig. $6 \mathrm{~g}$ ) and the second method involved breaking the function $f$ down into its component functions $u$ and $v$. Her second method avoided having to visualize a map from $\mathbf{R}^{2}$ to $\mathbf{R}^{2}$ because it allowed her to "think of two functions the real part going from $\mathbf{R}^{2}$ to $\mathbf{R}$ and the imaginary part going from $\mathbf{R}^{2}$ to $\mathbf{R}$. And .. f is continuous if and only if $u$ and $v$ are continuous" (Fig. 6h). Judy went on to say that she prefers to think about the value of $f$ as the height above the $x-y$ plane (Fig. 6i) and connected this perception to the discussion not lifting one's pencil off the page. She explained, "I might be trying to trace out the surface, trace out those height values and see if I can draw them. I'm not taking my pen off the page now, but I don't want to jump my pen anywhere" (Fig. 6j). Figure 7a-b depict Judy's IM of height and a surface.

This discussion triggered Judy's memory of a second IM metaphor that she uses to connect students' experiences of hiking to the CM notion of continuity of complexvalued functions (Fig. 7c). Judy explained:I don't want my pen to make any sudden precipitous drops. So, I often use a hiking analogy, especially in the classroom setting. Because the students are familiar with contour maps and falling off cliffs - not falling off cliffs; they tend to climb cliffs. So if I can draw this without a sudden change of altitude, then I'm continuous (Fig. 6k).

However, to make her idea more rigorous and to connect back to CM, Judy cautioned that one has to be able to "come in from any angle to do so," and that even then, there are examples where if one approaches a point along linear paths from all possible angles, the function still might be discontinuous at the point in question (Fig. 61-m). 


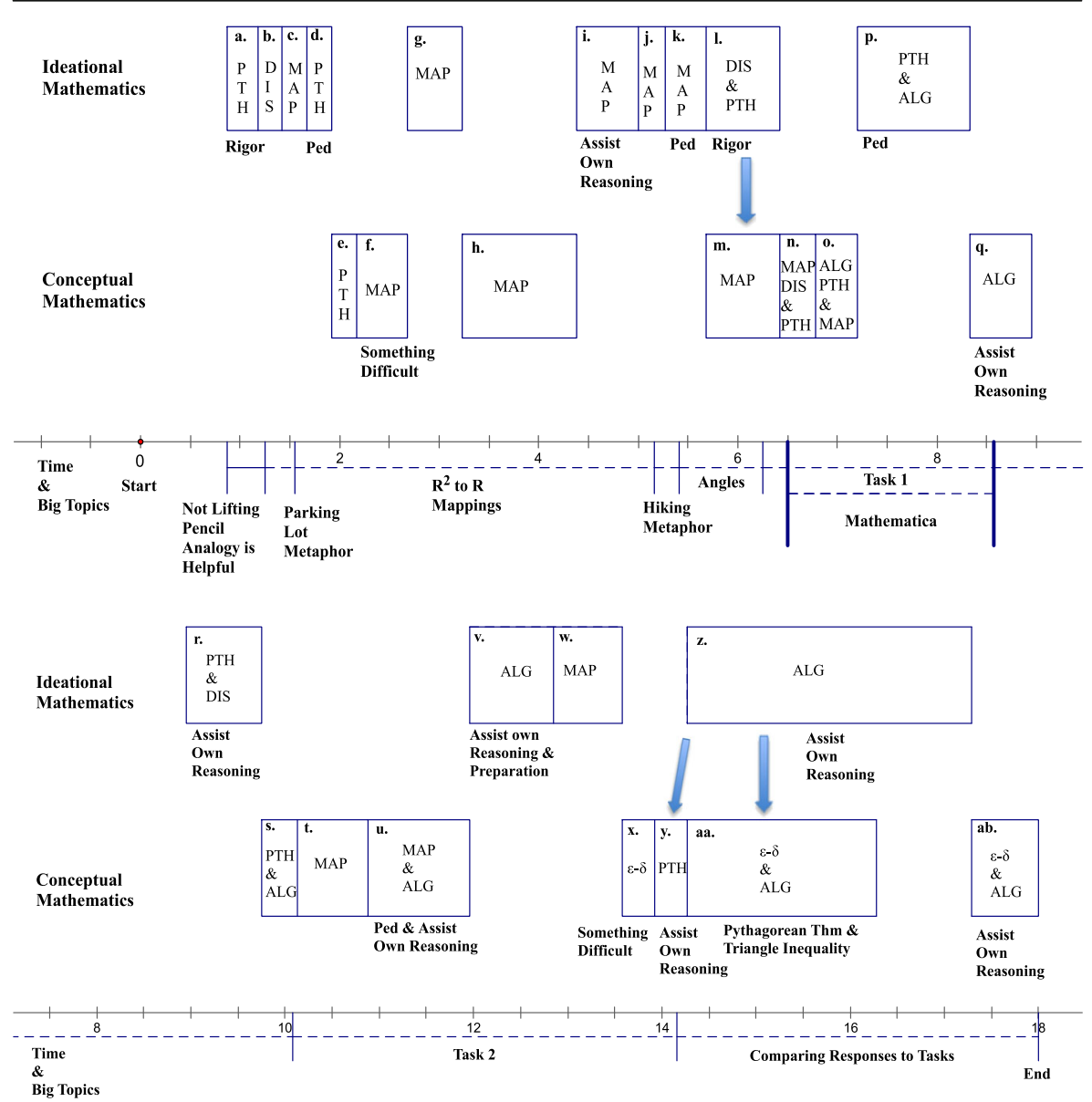

Fig 6 Judy's timeline

Judy continued to think about heights above the $x-y$ plane as she addressed the first task. She claimed it was not possible to make the function continuous at $x=0$ "because you can approach the origin from different directions and get different heights on the $x$ $y$ plane" (Fig. 6n). Similar to Becky, Judy integrated the Cartesian form and rewrote the function, $f(z)=\frac{\operatorname{Re}(z)}{|z|}$, as $\frac{x}{\sqrt{x^{2}+y^{2}}}$ and noted that one could obtain different function

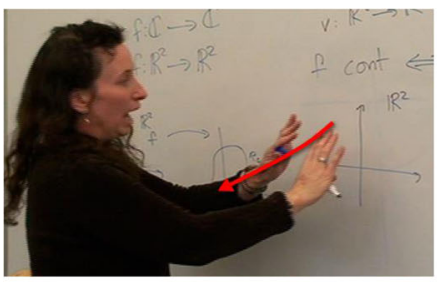

a) Representing the height

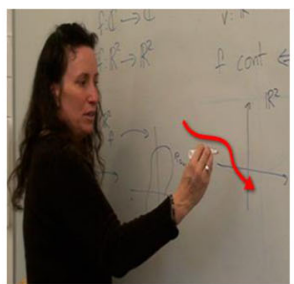

b) Tracing a surface

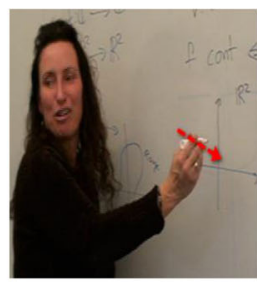

c) Depicting a sudden drop

Fig. 7 Judy's hiking metaphor 
values when approaching the origin from different directions, and hence there would be no way to get a "consistent height" (Fig. 6o). Taking a pedagogical perspective, Judy remarked that if she were showing this example to her class, she would display a twodimensional graph in Mathematica so that students could see that approaching the origin along the $y$-axis and along the $x$-axis yields different function values (Fig. 6p).

In response to the second task, Judy immediately recognized that it is possible to redefine the function to be zero at the origin and $f(z)=\frac{z \operatorname{Re}(z)}{|z|}$ elsewhere, so that this new function is continuous at the origin (Fig. 6t). She indicated that she would explain this to her students by writing the function in terms of its component functions, $f(z)$ $=\frac{z \operatorname{Re}(z)}{|z|}=\frac{(x, y) x}{\sqrt{x^{2}+y^{2}}}=\left(\frac{x^{2}}{\sqrt{x^{2}+y^{2}}}, \frac{x y}{\sqrt{x^{2}+y^{2}}}\right)$, each of which is continuous (Fig. 6u). However, she added that if she were to prove her claims, she would keep the function in terms of the variable $z$ because "there are obviously inequalities relating the real part to its modulus, which makes it easier to write an epsilon-delta proof" (Fig. 6v). Once again, Judy reflected how the Cartesian form allowed her to demonstrate to her students "lots of graphical examples" using Mathematica (Fig. 6w). She also mentioned that she realized that $f(z)=\frac{\operatorname{Re}(z)}{|z|}$ has "enough stuff on top to make it converge . . there are more $z$ 's on top to make it go to zero" (Fig. 6x-y).

Ultimately, Judy referenced the following CM notions throughout her interview: approaching along different paths, the graph of a function, mappings from one plane to another, discontinuity, real-valued component functions, contour maps, writing an "epsilon-delta proof," bounded functions, the triangle inequality, and convexity. Her IM language episodes included: the parking lot and hiking metaphors, hopping off the page and gaplessness, and viewing the component functions as the height above the $x-y$ plane. Generally, Judy invoked CM to support her IM statements and her IM language originated in order to assist her own reasoning, but she often connected these ideas to her teaching. For instance, Judy mentioned how she personally tends to think of the component functions $u(x, y)$ and $v(x, y)$ as heights above the $x-y$ plane and as a pedagogical tool she connected this idea to students' experience via the aforementioned hiking metaphor. The parking lot metaphor also allowed Judy to discuss the pedagogical transition from teaching limits in $\mathbf{R}$ to teaching limits in $\mathbf{C}$. Compared to the other participants, Judy discussed paths early on in her general description of continuity as well as with the two functions. Moreover, she discussed "mapping ideas" during her general description and with the two specific tasks.

\section{Luke}

Luke began his interview (Fig. 8) by discussing how the notion of drawing the graph of a function without lifting one's pencil is problematic when considering complex-valued functions because it is not possible to draw the graph of such functions (Fig. 8a-b). He claimed that this issue is compounded by the fact that students tend to treat a function and its graph as interchangeable (Fig. 8c). Accordingly, he mentioned several other common ways that mathematicians tend to visually convey complex-valued functions, such as a mapping from one plane to another or as a vector field (Fig. 8d). But 
according to Luke, neither of these other representations is "particularly enlightening" when it comes to assessing continuity. As a "fix," he proposed perceiving continuity in terms of "control" (Fig. 8f-i). He first stated this pedagogically in terms of preservation of closeness (Fig. 8j): "what I like to tell students about continuity is that small changes in the domain only cause small changes in the range." But immediately after this explanation, he cautioned:in order to do a mathematical definition you almost have to say that backwards, and you have to say 'give me a small change you are willing to live with in the range and I can guarantee you that I can find an area around this point $\left[z_{0}\right]$ where as long as you only pick values in here [in a disk centered at $\left[f\left(z_{0}\right)\right]$ that the function values that you get will stay inside here [in a disk centered at $z_{0}$ ].' It's the classic epsilon-delta explanation (Fig. 8k).

When asked to reconcile the informal IM language of preservation of closeness idea with his "mathematical" CM explanation, Luke expressed his control aspect in terms of an IM metaphor involving bombs on a target (Fig. 80-r). Essentially, once someone specifies an "acceptable" zone around the target (i.e., in the range), one can produce a region within the domain wherein any bombs launched from that region would be guaranteed to land in the acceptable target zone. Luke reiterated his language of control

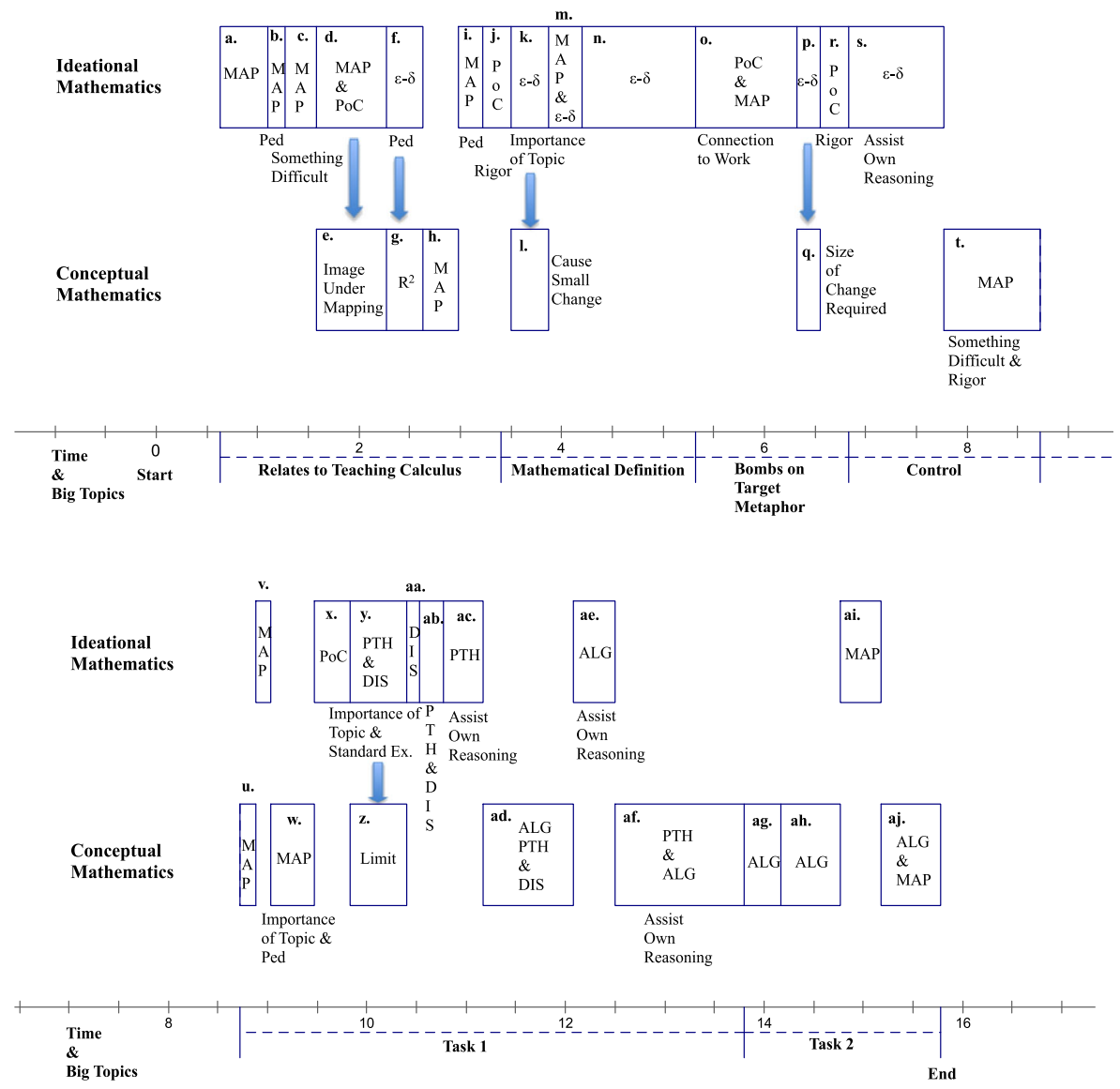

Fig. 8 Luke's timeline 
to separate it from the common understanding of merely causing small changes in the range as a result of small changes in the domain (Fig. 8s).

Speaking more mathematically, people like to informally say 'Oh continuity means small change in the domain gives you small change in the range' but that's really pretty poor because small is a meaningless term. You can have very small changes but have nothing to do with continuity. It really means ... you can control the changes in the range ... not that you get small changes in the range, but you can control how big the changes in the range are . . if I know how much change I'm willing to allow over there [in the range] then I can tell you what small change over here [in the domain] means.

Luke concluded by elaborating how the analogy of "not lifting your pencil" can be problematic because of the existence of functions which oscillate infinitely often so they cannot be drawn (with or without lifting your pencil) but are continuous and offered the diagram shown in Fig. 9.

In working with the first task, i.e. $f(z)=\frac{\operatorname{Re}(z)}{|z|}$, Luke immediately brought up the notion of a limit, as well as the requirement for continuity i.e. that $\lim _{z \rightarrow 0} f(z)=f(0)$ (Fig. 8u-w). Luke connected this idea to preservation of closeness as he said, "And, you know, in a large sense this captures this notion of closeness that we were talking about. You get close enough to zero, you'd better be exactly where [the function] was defined to be" (Fig. 8x-y). He related this limit discussion back to a more general notion from calculus, namely that for the limit to be unambiguously defined, you must get the same answer no matter which path you use to approach the point in question (in this case, $z=$ $0)$. He identified this notion as a "classic multivariable calculus issue" that did not necessarily have to do with properties of the complex numbers (Fig. 8z). Luke explained that if two paths of approach yielded different function values then the function is discontinuous (Fig. 8aa), and he related this notion back to his previous discussion about the requirement that $\lim _{z \rightarrow 0} f(z)=f(0)$ (Fig. 8ab). After simplifying the function to $\frac{x}{\sqrt{x^{2}+y^{2}}}$, Luke reiterated that this was basically a "calc III problem" and that this function would yield different values if one approached along the $x$-axis as opposed to the $y$-axis, and therefore is discontinuous at $z=0$ (Fig. 8ad). When asked why he chose to use the Cartesian form rather than the polar coordinates when working with the first example, Luke responded,

you gave me the real part of $z$ on the top, which forced me almost into rectangular coordinates. If I'm looking at a complex number in polar I don't know what the real

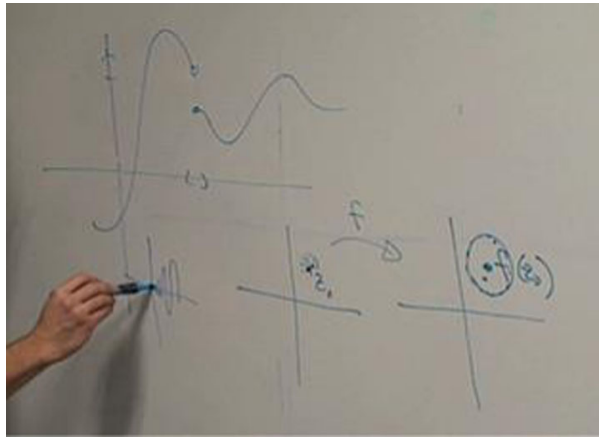

Fig. 9 Luke's illustration of a function that oscillates infinitely often so cannot be drawn, but is continuous 
part is and if I have an $x$ on the top then I probably want to deal with $x$ 's and $y$ 's on the bottom because that's algebraically more tractable (Fig. 8ae).

Luke added that considering the modulus of the entire function helped him to quickly determine that approaching zero along the $x$-axis and along the $y$-axis would yield different function values (Fig. 8af).

Considering the modulus of the function, $f(z)=\frac{\operatorname{Re}(z)}{|z|}$ allowed Luke to cancel $|z|$ in the numerator and denominator. He observed that,

because we're talking about this limit issue, you're not allowing $z$ to be zero yet, and so you just get absolute value of the real part (writes $|x|$ ). There's no denominator; there's no issue, because as I go to the origin this (points to $|x|)$ has got to go to zero. It doesn't matter how I get there (Fig. 8ag).

Luke elaborated how one could "fill in the hole" by defining the function to be zero at the origin (Fig. 8ah) since the limit of the function is zero. Finally, Luke mentioned that another way of thinking about the function is noticing that it essentially has a quadratic numerator and a linear denominator, so when considering the limit of this function, "it's all going to go to zero nicely" (Fig. 8aj).

Ultimately, Luke mentioned the following CM notions: the graph of a real-valued function, vector fields, continuity of a real-valued function, mapping from one plane to another, component functions of a complex-valued function, epsilon-delta definition, infinitely oscillating functions, continuity at a point, the limit of a complex-valued function, approaching the origin along different paths, and discontinuity. IM segments included the following topics: control, preservation of closeness, bombs on targets, and filling a hole. As mentioned above, Luke's IM notion of "control" was brought up as a way to address the absence of graphs from $\mathbf{C}$ to $\mathbf{C}$. But in order to make this control idea rigorous he discussed how preservation of closeness ideas could be modified to incorporate more CM in the "classical epsilon-delta explanation." Although Luke's general discussion about continuity primarily consisted of ideas related to mapping, preservation of closeness, graphs, and control, his discussion related to the tasks contained path and limit-related ideas.

\section{Rafael}

Rafael's timeline is shown in Fig. 10. Rafael began his explanation by considering the perspective and needs of students. He mentioned how he tried to convey the notion of "functions as deformations of physical space" which prepare students to think about higher dimensions. In particular, he felt this allowed him to introduce the notion of mappings that take a point $z_{0}$ and map it to another point $w_{0}$. As with most of our participants, Rafael first discussed continuity in terms of preservation of closeness, explaining, "that in the small scale setting, our nearby points map to nearby points" (Fig. 10a-e). He expressed a need to formalize this notion, and "put a rigorous definition or description on that." Curiously, instead of connecting the IM notion of preservation of closeness to the standard CM "epsilon-delta" definition, he discussed an IM metaphor of a bulls-eye target (Fig. 10f-k).

... imagine that we draw a bulls eye target ... we know that this point $\left[z_{0}\right]$ is going to hit dead center. The question is if we're close enough to this point $\left[z_{0}\right]$, will all the points in here [a disk centered around $z_{0}$ ] end up in the target [points to disk about $w_{0}$ ]? 


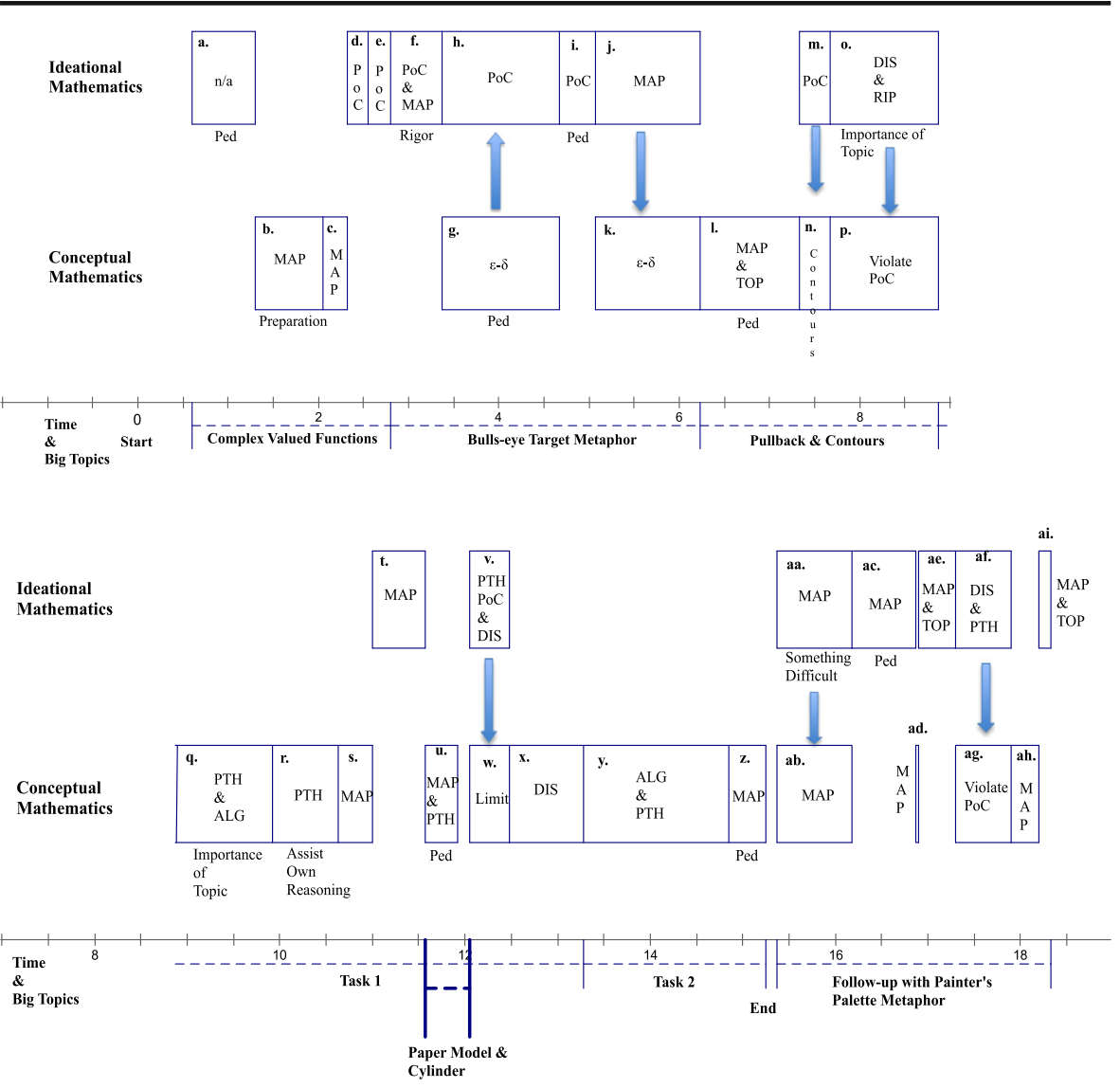

Fig. 10 Rafael's timeline

... If I specify the size here [the codomain], does some disk centered at $z_{0}$ map to the bulls-eye zone?

Rafael's bulls eye example made only tenuous connections to the formal mathematical definition of continuity, thus we asked him to elaborate the connection. Rafael explained how he could adopt the notion of a pull-back, that is, "work backwards and draw the contour map ... so look at the inverse image to pull back" (Fig. 101-p). He clarified how the contour levels measure "how close you are to your target" and that it is important "to make sure that a whole chunk of space over here [in domain] gets mapped to that zone [in co-domain]." Rafael went on to explain the importance of considering discontinuous maps, which he described as a mapping "that takes a path to the plane, splits it apart and moves two points that were nearby far apart." He demonstrated this by actually tearing a piece of paper as shown in Fig. 11. As such, he used IM to convey how a discontinuous map violates preservation of closeness.

Rafael spent quite a bit of time reasoning through the first task (10q-x) using CM notions, which he felt were important, assisted his own reasoning, or helped convey ideas to students. He explained how classical examples such as $f(z)=\frac{\operatorname{Re}(z)}{|z|}$ are "designed to be analyzed in polar coordinates and the basic idea for a lot of these 


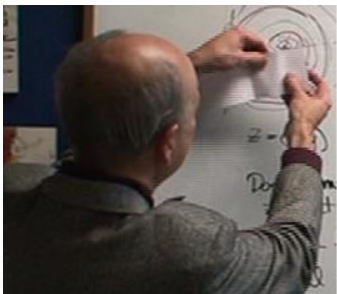

a) Representing paper as region

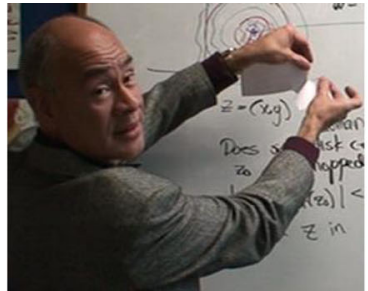

b) Tearing paper

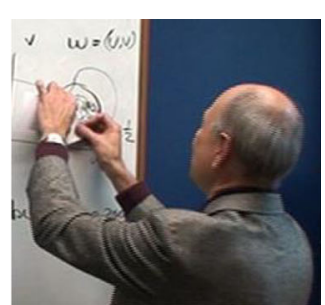

c) Showing image of paper

Fig. 11 Rafael's discontinuity and tearing paper

examples is that if you vary the direction of approach you get different values." Rafael further explained how polar coordinates allow one to conveniently determine the continuity at a point as he wrote: $\frac{x}{r}=\frac{r \cos \theta}{r}=\cos \theta$, which he explained depended on theta instead of the radius. After showing that the function results in different values for theta equal to 0 and $\frac{\pi}{2}$, Rafael mentioned that one can "graph it in the traditional three dimensional coordinates." In fact, he graphed a cosine wave on a thermos to further illustrate how the "heights are different so there's not a single value that you can assign that works in the whole neighborhood." Thus, he concluded that it was not possible to "define a value at the origin that would make the function continuous."

Rafael returned to the polar form as he worked with the second function, $f(z)$ $=\frac{z \operatorname{Re}(z)}{|z|}$ and once again adopted CM (Fig. 10y-z). He rewrote the function as $f(z)$ $=\frac{z R \mathbf{e}(z)}{|z|}=\frac{r e^{i \theta} r \cos \theta}{r}=r e^{i \theta} \cos \theta$ and remarked that $e^{i \theta} \cos \theta$ is bounded and as $r$ goes to zero, the entire function goes to zero. He added, "in fact this is uniform convergence to zero in every direction." Rafael concluded that although he wouldn't mention it to students, the function is a Lipschitz function "so we can define $f$ to be zero [at zero] and then it will be continuous."

In a follow-up interview, Rafael once again attempted to reconcile informal preservation of closeness ideas with the formal $\mathrm{CM}$ definition of continuity through another IM metaphor (Fig. 10aa-ai). He mentioned that because it is impossible to draw graphs due to the dimensionality issue one has to work "harder" to convey the continuity notion.

... take an artist's palette of colors here [in the codomain] (Fig. 12a) and we're going to do a paint by number scheme [...] So this $[f]$ is a mapping; it's an instruction that tells you for every point [in the domain] (Fig. 12b) how to color it. [...] And an example of a discontinuous function would be where at a given point [in the domain], you have dramatically different colors all coming together at this one point (Fig. 12c).

In this case, Rafael incorporated IM language to convey CM notions related to mappings and discontinuity as he stated, "I call this pulling back the function."

Generally speaking, Rafael's use of CM heavily relied on mapping-related language, ideas, and examples. Mapping-related topics included the point-wise behavior of a mapping, a mapping from a plane to another plane, and the graph of a complex-valued function. A common manifestation of Rafael's reliance on mappings is that of a pullback, whether it was pulling back the image of contour curves, or when it was used more implicitly in his bulls-eye target and paint-by-color scheme IM metaphors. Like 


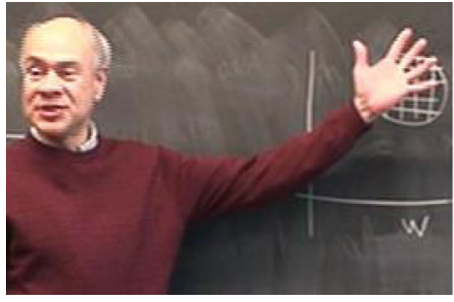

a) Holding artist's palette of colors

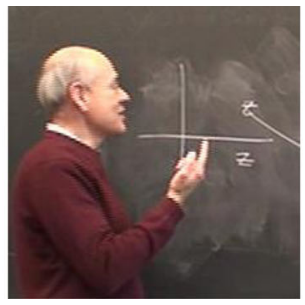

b) Mapping points

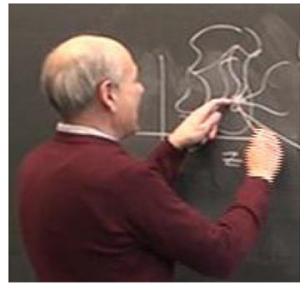

c) Illustrating discontinuity

Fig. 12 Rafael's artist's palette metaphor

other participants, Rafael introduced discontinuity on his own, and used "tearing" or "splitting apart" language to connect intuitive physical processes and the IM preservation of closeness to the CM idea of discontinuity. Moreover, several statements appeared to have a pedagogical intention.

\section{Discussion and Implications}

We observed that the mathematicians offered IM notions addressing the domain first rather than the codomain first, as found with the CM definition continuity. Some of these IM notions intertwined with CM notions, served an intentional purpose, and appeared to stem from the participants' embodied physical experiences. In this section, we summarize these findings and offer teaching implications, which may serve as directions for future research.

\section{Mathematicians' IM Notions}

There were four IM notions that the mathematicians used to convey the idea of continuity for complex-valued functions: control, physical embodied experiences attempting to capture topological features, preservation of closeness, and paths. These notions were attempts to portray the $\mathrm{CM} \varepsilon-\delta$ definition of continuity, but only the IM involving control and topological notions explicitly expressed the structure of this definition. Following the analysis of Oehrtman et al. (2014), the formal definition of continuity consists of three quantifiers (for the variables $\varepsilon, \delta$, and $z$, which represent quantities associated with the codomain, domain, and domain, respectively) followed by a predicate on quantities in the codomain. In this sense the definition is a codomaindomain-domain-codomain statement. Previous research identifying students' tendency toward domain-first reasoning for limits (Cottrill et al. 1996; Swinyard and Larsen 2012) refers to the final domain-codomain pair; given $z$ in the domain, one examines the distance between $f(z)$ and $f\left(z_{0}\right)$ in the codomain. Codomain-first reasoning refers to the initial codomain-domain pair of quantifiers for $\varepsilon$ and $\delta$, the dependence of $\delta$ on $\varepsilon$, and their roles in framing the remainder of the definition.

Although none of the participants explicitly stated the formal definition of continuity, episodes designated with the $\varepsilon-\delta$ code were the closest responses to doing so. The participants who mentioned explicitly or implicitly that they were trying to find a $\delta$ that "works" for a given $\varepsilon$ did so by introducing the notion of control. This codomain-first 
IM concept first expresses $\varepsilon$ as a tolerance in the codomain, and then $\delta$ as the means of control in the domain to achieve that tolerance. Topological properties of continuous complex-valued functions were also frequently used to express codomain-first concepts. For example, Rafael's pullback explanation of the bulls-eye target used the distance to $z_{0}$ as a way to generate level curves in the domain which he later refined as a pull-back of colors on a painter's palette in the codomain. The recognition of discontinuity was then described as "dramatically different colors," expressing a counterexample $\varepsilon$, "all coming together at this one point," expressing a failure of the remainder of the definition for all $\delta$. Although Andrew's CM topological characterization of continuous functions in terms of the preimage of open sets being open does move from a statement about the codomain to one about the domain, he did not explicitly use the metric to translate this condition to a codomain-first conceptualization of the $\varepsilon-\delta$ definition.

An IM notion that the participants expressed as a domain-first concept was preservation of closeness: points that are "close" in the domain are mapped to points that are "close" in the codomain. As Luke expressed, "closeness" in such a formulation is vague and requires the codomain-first part of the definition to make it rigorous. This domain-first conceptualization of preservation of closeness provided the basis for nearly all participants' intuitive explanation of general continuity. This explanation commonly coincided with IM verbiage such as "nearby points map to nearby points" which transformed into a discussion about discontinuity. Participants often expressed discontinuity as a violation of some version of preservation of closeness. Becky used an image of a rip in a sheet of silly putty to describe a violation of preservation of connectedness (e.g., a line through the discontinuity is mapped to separated components, or ripped). All of the experts described some discontinuity as a domain-first violation of preservation of closeness by sending nearby points (in the domain) far away from each other (in the codomain).

Another domain-first conceptualization of continuity was that of reaching the same limit when approaching a point, in the domain through various paths. This reasoning was prevalent when the participants explored the task functions for continuity and remained central to their demonstration for the discontinuous function since only two paths with different limits need be considered. Several of the experts considered multiple types of paths as they explored the continuous example, some doing so to the point of convincing themselves of continuity. For example, Luke concluded, "as I go to the origin this $[|x|]$ has got to go to zero. It doesn't matter how I get there," and Rafael made a similar argument. However, for the continuous function, Andrew, Becky, and Judy shifted to an argument of bounding $|f(z)|$ as if moving toward an $\varepsilon-\delta$ proof, although none articulated a reason for the shift (e.g., it is not possible to account for all possible paths).

As in Núñez and Lakoff's (1998) notion of "natural continuity," Luke and Judy both superimposed a description of motion in their explanation of approaching a point along multiple paths. Noting that continuity of a complex-valued function is equivalent to continuity in each component, Judy portrayed the graph of a function from $\mathbf{R}^{2}$ to $\mathbf{R}$ as a landscape one might hike on, and a discontinuity as falling off of a cliff. As in Oehrtman's (2009) study of students' reasoning about continuity of multivariable functions, such motion is added to an image of a landscape in which the discontinuity may be conceived of without the motion, as in the presence of a cliff. Luke noted that 
motion imagery such as drawing a graph without lifting a pencil is suggestive but may be misleading because functions such as the one shown in Fig. 9 are continuous but may not be traversed as the path of some physical motion. Núñez and Lakoff note that such images of "natural continuity" conflict with formalized versions of continuity as seen by the distinction in Luke's drawing.

\section{Interplay Between IM and CM}

While we coded episodes as primarily IM or primarily CM, we quickly recognized that rarely was a statement completely IM or completely $\mathrm{CM}$. Indeed, many statements made by participants were referential to formal CM concepts, but were expressed through IM language. This influenced our decision to include arrows in participants' timelines for such situations, depicting what we came to informally refer to as "nearCM" statements. Though the interplay between IM and CM was not a central focus of Schiralli and Sinclair's (2003) framework, our study has demonstrated that these two constructs interweave frequently as part of a larger continuum between informally personal IM and formally negotiated CM. In studying the timelines, one observes the interplay between CM and IM and the purposeful ways in which the mathematicians introduced the notions. Generally, the participants invoked IM for pedagogical purposes, in preparation for other explanations, and to assist in their reasoning. The IM portrayed was offered as an effort to develop students' IM, such as with Judy's parking lot and hiking examples, as well as to support students' CM, such as with Rafael's painter's palette example. The mathematicians occasionally invoked CM to prepare for another action; for instance, Judy used Cartesian coordinates to prepare for entering expressions into Mathematica, which she used for pedagogical purposes.

The mathematicians also invoked IM as a mechanism to assist their reasoning about CM notions, especially to ensure that their IM contained the structure of the CM analog (i.e., as a scientific model, Vygotsky 1987). For instance, Rafael's painter's palette subsumed the structure of a pullback, while Judy's hiking metaphor was designed for students to think about the characteristics of the real and imaginary components of a complex-valued function. Furthermore, the participants often instantiated preservation of closeness by discussing "control" or "ripping" for the purpose of illustrating aspects of the formal epsilon-delta definition of continuity that were either upheld or violated. It is worth noting that as Schiralli and Sinclair (2003) indicated, each mathematician's IM stems from his/her own embodied experiences including embodied CM. For instance, childhood memories of silly putty and paint-by-numbers influenced Becky and Rafael's IM. Judy's IM was influenced by her own driving and hiking experiences while Luke's military knowledge influenced his bombs on targets IM metaphor.

On the other hand, mathematicians typically invoked CM when they did not perceive their IM language or examples to be "rigorous" or "mathematical" enough. Moreover, no participant articulated a clear link between the IM notion of preservation of closeness and the formal epsilon-delta definition of continuity, even when explicitly asked to do so. Most mathematicians invoked CM that they believed was most powerful to serve a particular purpose. For instance, using polar coordinates made it easier for some participants to algebraically simplify particular examples. Additionally, considering different paths of approach allowed some participants to address discontinuity with ease. 
It is vital to note that our participants were mathematicians, and accordingly have highly developed and integrated spontaneous and scientific concepts (Vygotsky 1987). As such, the interview discussions were not in the participants' ZPD, as nearly all aspects of their reasoning were under conscious awareness and volitional control. Their responses were highly structured, with clear connections made explicit between intuitive and formal reasoning. However, the nature of these mathematicians' CM and IM reflected the roles we would expect from their developmental strengths and weaknesses. Specifically, participants used IM to ground ideas in concrete meaning and interpretation, to give them immediate and intuitive access to basic properties and relationships. On the other hand, they referenced CM to make rigorous arguments, elaborate specific examples, and connect to broader content. As anticipated, their CM reflected the mathematics community norms and practices, while their IM was often personal and occasionally idiosyncratic.

\section{Teaching Implications and Future Research}

One of the most surprising results of our study was the fact that no participant successfully articulated an explicit link between their informal preservation of closeness IM and the formal CM epsilon-delta definition of continuity, even when explicitly asked to do so. Given that nearly all of these participants mentioned that they include both preservation of closeness intuition and formal epsilon-delta arguments in their teaching, a natural question arises: How can instructors stress the interplay between CM and IM as they teach a mathematical concept such as continuity of complex-valued functions? While our results do not provide an answer to this question, it seems that this issue at least warrants additional consideration by teachers. In particular, while incorporating IM into mathematics instruction can be beneficial to students' development, it seems that instructors should be careful to be purposeful in stating how their IM relates to the formal structure of the referenced CM notions. We caution instructors to be explicit in pointing out and providing specific reasons why an IM metaphor does not fully capture the missing formal CM properties, especially when an IM metaphor is designed to build intuition of a CM concept. Modeling such deconstruction and coordination with students may help them recognize the various aspects of mathematical objects and how these pieces "can be brought together to serve as a generative role in the testing and generalization of mathematical relationships" (Wilkerson-Jerde and Wilensky 2011, p. 40).

Another related question that arises from this study is, how might students be able to develop a relationship between IM and CM while in their ZPD? To facilitate this type of exploration, instructors might design activities based on their own IM/CM interplay or wherein students formulate their own IM and compare this intuition to formal CM definitions. Given that the mathematicians conveyed IM that appeared to stem from physical embodied experiences, we hypothesize that students can similarly craft IM by reflecting on their own embodied experiences. We encourage further research on deliberately mediating this type of student reflection and developing explicit reasons for invoking IM and CM (see Table 2 in Appendix) in classroom discussions. Such efforts could lead to second-generation research, where one adopts "advances in educational psychology and cognitive science to inspire changes in course design" (Freeman et al. 2015, p. 8412). 
Because CM tends to strip away some of the more informal aspects of IM in the interest of being rigorous and unambiguous, and because CM tends to be more explicitly valued in classroom assessments than IM, students might believe that IM has no place in the mathematics classroom, or that it is not necessary beyond a certain point in their understanding. However, our research suggests that mathematicians not only invoke IM for pedagogical purposes, but also to assist their own reasoning. If instructors can be more deliberate about conveying the importance of IM, perhaps students can learn to explore how IM and CM can complement one another. Such teaching allows students to connect the new mathematics to their own embodied experiences including their embodied CM, which is in contrast to teaching that values a polished product as that found in a textbook. Given that some participants mentioned how they prefer to incorporate technology into their instruction, another question that naturally arises is how can instructors utilize technology in the classroom to facilitate the development of students' IM and clarify the relationship between that IM and CM concepts? All of these teaching suggestions open the door to investigate students' interplay of CM and IM of continuity of complex-valued functions.

\section{Appendix}

\section{Coding Scheme}

Table $1 \mathrm{IM} / \mathrm{CM}$ codes and their corresponding descriptions

\begin{tabular}{|c|c|}
\hline Code & Description/Criteria/Examples \\
\hline $\begin{array}{l}\text { Preservation of } \\
\text { Closeness (PoC) }\end{array}$ & $\begin{array}{l}\text { A "domain-first" conceptualization of continuity in which closeness in the domain } \\
\text { yields closeness in the range. Examples: Close goes to close, referencing disks, } \\
\text { balls, open sets, images of these, neighborhoods, chunks of space/blobs }\end{array}$ \\
\hline Mapping (MAP) & $\begin{array}{l}\text { Discussion of a mapping (complex or otherwise) and its accompanying properties. } \\
\text { Examples: mapping a plane to a plane or point-wise, pre-image of sets, splitting } \\
\text { up a complex valued function into its real and imaginary components }\end{array}$ \\
\hline Topological (TOP) & $\begin{array}{l}\text { Participant used a topological property to communicate an idea. Examples included } \\
\text { pullbacks and the inverse image of a function preserving openness of sets (i.e., } \\
\text { topological definition of continuity). }\end{array}$ \\
\hline Paths (PTH) & $\begin{array}{l}\text { Participant discussed approaching a point via different paths or directions, usually } \\
\text { in conjunction with discussion of limits. }\end{array}$ \\
\hline Ripping (RIP) & $\begin{array}{l}\text { Participant referenced ripping or tearing an object. Usually occurred when } \\
\text { discussing discontinuity, as a violation of PoC. }\end{array}$ \\
\hline Discontinuity (DIS) & Explicit mention of discontinuity of a function. \\
\hline Epsilon-Delta $(\varepsilon-\delta)$ & $\begin{array}{l}\text { Participant expressed a concern for quantification related to continuity. In particular, } \\
\text { the participant referenced a specific delta that "works" for a given epsilon. } \\
\text { Primary example was "control." }\end{array}$ \\
\hline
\end{tabular}

Algebraic Work (ALG) Participant discussed algebraic manipulation of a function or a preference for a given algebraic representation of a function (for instance, polar or Cartesian) 
Table 2 Reason for using CM or IM and their corresponding descriptions

\begin{tabular}{|c|c|}
\hline Code & Description \\
\hline Importance of Topic & $\begin{array}{l}\text { Participant identified a particular notion or subject as important to the } \\
\text { mathematical community. }\end{array}$ \\
\hline Pedagogical (Ped) & $\begin{array}{l}\text { Participant brought up a certain topic or way of thinking for } \\
\text { pedagogical purposes. Examples include connecting a } \\
\text { mathematical concept with student experience, providing new ways } \\
\text { to think about a familiar topic, and communicating ideas in ways } \\
\text { students would be able to understand. }\end{array}$ \\
\hline Assist Own Reasoning & $\begin{array}{l}\text { Participant discussed how thinking of a particular concept or task in a } \\
\text { certain way is more personally tractable. }\end{array}$ \\
\hline Rigor & $\begin{array}{l}\text { Participant described a mathematical concept in a particular way in } \\
\text { order to be rigorous or comprehensive. }\end{array}$ \\
\hline $\begin{array}{l}\text { Connection to Own Work/Research } \\
\text { (Connection to Work) }\end{array}$ & $\begin{array}{l}\text { Participant thought about or brought up a certain topic because it is } \\
\text { applicable to his or her own work. }\end{array}$ \\
\hline $\begin{array}{l}\text { Something is Difficult (Something } \\
\text { Difficult) }\end{array}$ & $\begin{array}{l}\text { Participant introduced a new topic or way of thinking about a topic to } \\
\text { ameliorate an inherently difficult situation. For instance, } \\
\text { participants discussed alternative ways to visualize complex valued } \\
\text { functions given that one cannot graph these functions in the } \\
\text { traditional sense. }\end{array}$ \\
\hline Convention & $\begin{array}{l}\text { Participant brought up a particular topic or chooses a particular } \\
\text { representation of a topic to align with mathematical convention. }\end{array}$ \\
\hline $\begin{array}{l}\text { Preparation for Another Purpose } \\
\text { (Preparation) }\end{array}$ & $\begin{array}{l}\text { Participant discussed a particular topic as preparation for another } \\
\text { purpose. Examples included expressing a function in Cartesian } \\
\text { form to later input it into a CAS, or performing algebraic } \\
\text { manipulations for the purpose of later visualizing the problem } \\
\text { geometrically. }\end{array}$ \\
\hline
\end{tabular}

\section{References}

Anderson, K. C., \& Leinhardt, G. (2002). Maps as representations: Expert novice comparison of projection understanding. Cognition and Instruction, 20(3), 283-321.

Black, M. (1962). Metaphor. In M. Black (Ed.), Models and metaphors: Studies in language and philosophy (pp. 25-47). Ithaca: Cornell University Press.

Black, M. (1977). More about metaphor. Dialectica, 31, 433-457.

Brown, J. W., \& Churchill, R. V. (2009). Complex variables and applications. Boston: McGraw-Hill.

Châtelet, G. (2000). Figuring space: Philosophy, mathematics, and physics. Translated by R. Shore and M. Zagha. Dordrecht: Kluwer Academic Publishers.

Cottrill, J., Dubinsky, E., Nichols, D., Schwingendorf, K., Thomas, K., \& Vidakovic, D. (1996). Understanding the limit concept: Beginning with a coordinate process schema. Journal of Mathematical Behavior, 15, 167-192.

Danenhower, P. (2000). Teaching and learning complex analysis at two-British Columbia universities. Dissertation Abstract International 62(09). (Publication Number 304667901). Retrieved March 5, 2011 from ProQuest Dissertations and Theses database.

Danenhower, P. (2006). Introductory complex analysis at two British Columbia universities: The first week complex numbers. In F. Hitt, G. Harel, \& A. Selden (Eds.), Research in collegiate mathematics education VI. Rhode Island: AMS.

de Freitas, E., \& Sinclair, N. (2012). Diagram, gesture, agency: Theorizing embodiment in the mathematics classroom. Educational Studies in Mathematics, 80(1-2), 133-152. 
Fisher, B. (2010). Conceptualizing Multivariable Limits: From Paths to Neighborhoods. Proceedings for the Thirteenth SIGMAA on Research in Undergraduate Mathematics Education Conference. Raleigh, NC: North Carolina State University, 20 pages, Web publication at http://sigmaa.maa.org/rume/crume2010 /Archive/Fisher.pdf.

Freeman, S., Eddy, S. L., McDonough, M., Smith, M. K., Okoroafor, N., Jordt, H., \& Wenderoth, M. P. (2015). Active learning increases student performance in science, engineering, and mathematics. Proceedings of the National Academy of Sciences, 11(23), 8410-8415.

Harel, G. (2013). DNR-based curricula: The case of complex numbers. Journal of Humanistic Mathematics, 3(2), 2-61.

Inglis, M., \& Alcock, L. (2012). Expert and novice approaches to reading mathematical proofs. Journal for Research in Mathematics Education, 43(4), 358-390.

Lakoff, G., \& Núñez, R. E. (2000). Where mathematics comes from: How the embodied mind brings mathematics into being. New York: Basic Books.

Martin, J. (2013). Differences between mathematicians' and students' conceptual images of the mathematical structure of Taylor series convergence. Educational Studies in Mathematics, 82, 267-283.

Nemirovky, R., Rasmussen, C., Sweeney, G., \& Wawro, M. (2012). When the classroom floor becomes the complex plane: Addition and multiplication as ways of bodily navigation. Journal for the Learning Sciences, 21(2), 287-323.

Nemirovsky, R. \& Smith, M. (2011). The physicality of symbol use: Projecting horizons and traversing improvisational paths across inscriptions and notations: In (Eds.) S. Brown, S. Larsen, K. Marrongelle, \& M. Oehrtman, Proceedings of the 14th Annual Conference on Research in Undergraduate Mathematics Education, Vol. 3, pp. 314-324. Portland.

Núñez, R. E., \& Lakoff, G. (1998). What did Weierstrass really define? The cognitive structure of natural and $\varepsilon-\delta$ continuity. Mathematical Cognition, 4(2), 85-101.

Oehrtman, M. (2009). Collapsing dimensions, physical limitation, and other student metaphors for limit concepts. Journal for Research in Mathematics Education, 40, 396-426.

Oehrtman, M., Swinyard, C., \& Martin, J. (2014). Problems and solutions in students' reinvention of a definition for sequence convergence. Journal of Mathematical Behavior, 33(1), 131-148.

Panaoura, A., Elia, I., Gagatsis, A., \& Giatlilis, G. P. (2006). Geometric and algebraic approaches in the concept of complex numbers. International Journal of Mathematical Education in Science and Technology, 37(6), 681-706.

Schiralli, M., \& Sinclair, N. (2003). A constructivist response to 'Where mathematics comes from.'. Educational Studies in Mathematics, 52(1), 79-91.

Sinclair, N., \& Tabaghi, S. G. (2010). Drawing space: Mathematician’s kinetic conceptions of eigenvectors. Educational Studies in Mathematics, 74, 223-240.

Soto-Johnson, H. (2014). Visualizing the Arithmetic of Complex Numbers. International Journal for Technology in Mathematics Education, 21(3), 103-114.

Soto-Johnson, H., \& Troup, J. (2014). Reasoning on the complex plane via inscriptions and gesture. The Journal of Mathematical Behavior, 36, 109-125.

Swinyard, C., \& Larsen, S. (2012). What does it mean to understand the formal definition of limit?: Insights gained from engaging students in reinvention. Journal for Research in Mathematics Education, 43(4), 465-493.

Tall, D., \& Vinner, S. (1981). Concept image and concept definition in mathematics with particular reference to limits and continuity. Educational Studies in Mathematics, 12, 151-169.

Talmy, L. (1996). Fictive motion in language and "caption.". In P. Bloom, M. Peterson, L. Nadel, \& M. Garrett (Eds.), Language and space. Cambridge: MIT Press.

Vygotsky, L. (1987). The development of scientific concepts in childhood. In R. W. Rieber \& A. S. Carton (eds.) The collected works of L.S. Vygotsky. Vol. 1, 167-241. (Original work published in 1934).

Weber, K. (2008). How mathematicians determine if an argument is a valid proof. Journal for Research in Mathematics Education, 39(4), 431-459.

Weber, K., \& Mejia-Ramos, J. P. (2011). Why and how mathematicians read proofs: An exploratory study. Educational Studies in Mathematics, 76, 329-344.

Wilkerson-Jerde, M. H., \& Wilensky, U. J. (2011). How do mathematicians learn math?: Resources and acts for constructing and understanding mathematics. Educational Studies in Mathematics, 78, 21-43.

Williams, S. (1991). Models of limit held by college calculus students. Journal for Research in Mathematics Education, 22, 219-236. 